\title{
Étude théorique et expérimentale d'une source EUV par décharge capillaire
}

\author{
J. Pons, K. Lan, C. Cachoncinlle, E. Robert, R. Viladrosa, \\ J.M. Pouvesle et C. Fleurier
}

GREMI, Université d'Orléans, CNRS, 14 rue d'Issoudun, BP. 6744, 45067 Orléans cedex 2, France

\begin{abstract}
Résumé : le plasma engendré par une décharge capillaire rapide à haute tension en vue de la production de rayonnement extrême-ultraviolet (EUV) est étudié expérimentalement par imagerie sténopé résolue en temps. L'évolution temporelle du profil radial d'émission dans différentes bandes spectrales est déduite des mesures, et simulée au moyen d'un code de calcul spécifique combinant les phénomènes magnétohydrodynamiques et la physique atomique. Les résultats montrent la formation du plasma sur l'axe du capillaire, initiée par un faisceau d'électrons résultant de l'effet de cathode creuse, puis une rapide compression suivie d'une expansion radiale. La densité électronique a été estimée d'après le code à environ $4.10^{17} \mathrm{~cm}^{-3}$ avec un profil radial convexe.
\end{abstract}

\section{INTRODUCTION}

Un circuit de type Blumlein permettant d'engendrer des impulsions de fort courant $(9-15 \mathrm{kA})$ de faible durée (demi-période $120 \mathrm{~ns}$ ) dans un capillaire de longueur $5 \mathrm{~cm}$ et de diamètre $1,5 \mathrm{~mm}$, est utilisé pour produire des plasmas de fortes température et densité électroniques émettant intensément dans le domaine extrême-ultraviolet (EUV, 5-100 nm). L'objet de ce système est d'obtenir une émission cohérente de la raie à $46,9 \mathrm{~nm}$ émise par l'ion $\mathrm{Ar}^{8+}$ selon un schéma de pompage par excitation [1], en utilisant un procédé différent des dispositifs par décharge capillaire utilisés jusqu'à présent [2-5]. Ce système est compact et fonctionne avec une faible énergie injectée $(10-30 \mathrm{~J})$; la forme des électrodes du capillaire a en outre été modifiée par rapport à une précédente configuration [6], afin de favoriser l'effet de cathode creuse [7] qui assure l'initiation du plasma. Selon ce procédé, un faisceau d'électrons provenant de la cathode est injecté dans le capillaire et provoque l'ionisation du gaz [8].

L'étude menée ici a pour but d'étudier l'évolution temporelle du profil radial d'émission du plasma, dans différentes gammes spectrales du domaine EUV, afin de déterminer la dynamique du plasma et d'en déduire si un tel système permet ou non d'obtenir l'effet laser recherché. En effet, pour que l'inversion de population ait lieu, il est nécessaire de réunir des conditions particulières, à savoir $\mathrm{T}_{\mathrm{e}} \sim 60 \mathrm{eV}$ et $\mathrm{N}_{\mathrm{e}} \sim 5.10^{18} \mathrm{~cm}^{-3}$ avec un profil radial concave [3,9]. Les expériences correspondantes sont réalisées par imagerie sténopé résolue en temps.

Le circuit est constitué de deux condensateurs plans rectangulaires $(1,5 \times 1 \mathrm{~m})$ possédant une armature commune et d'un commutateur de type éclateur atmosphérique, dont l'arc est guidé entre ses électrodes par une surface diélectrique (figure 1a). La tension appliquée pour charger les condensateurs varie entre 15 et $25 \mathrm{kV}$. La décharge s'effectue dans l'argon injecté à une pression de $1 \mathrm{mbar}$ dans un capillaire constitué d'alumine $\left(\mathrm{Al}_{2} \mathrm{O}_{3}\right)$. La figure $1 \mathrm{~b}$ montre le courant engendré dans le capillaire pour une tension de claquage de $-20 \mathrm{kV}$.

Un code de calcul lagrangien à une dimension [10] est employé pour la simulation des profils radiaux d'émission aux différents instants. Il inclut les phénomènes magnétohydrodynamiques et la physique atomique, et prend en compte le profil radial de la densité de courant comme paramètre, afin de respecter la symétrie du plasma observé.

*Adresse permanente: Institute of Applied Physics and Conmutational Mathematics, P.O. Box 8009-12, Beijing 100088, Chine. 


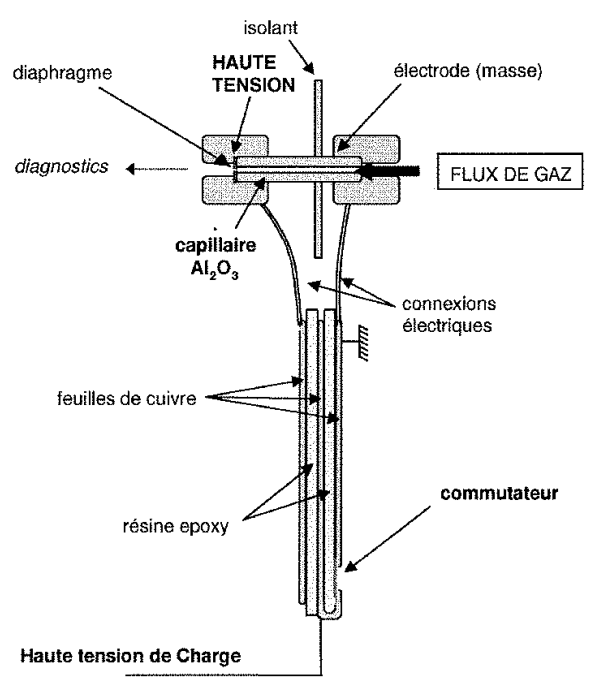

(a)

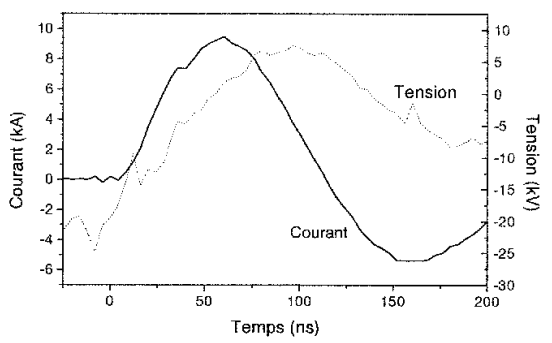

(b)

Figure 1 : (a) Circuit Blumlein de décharge - (b) Courant de décharge et tension aux bornes du capillaire

\section{RÉSULTATS EXPÉRIMENTAUX}

Les mesures ont été effectuées pour une tension de claquage dans le capillaire égale à $-20 \mathrm{kV}$, qui engendre un courant de $9 \mathrm{kA}$ d'amplitude maximale avec une vitesse de montée de $0,2 \mathrm{kA} / \mathrm{ns}$ (figure $1 \mathrm{~b}$ ). Le diaphragme utilisé pour l'imagerie mesure $50 \mu \mathrm{m}$ de diamètre, et est disposé de manière à obtenir un grandissement égal à 6,5. Les figures ci-dessous (figures $2 \mathrm{a}$ et 2b) montrent les images acquises pour différents domaines de longueurs d'onde, et les profils radiaux qui en sont déduits. La première série correspond à l'émission entre 5 et $200 \mathrm{~nm}$ (domaine de sensibilité du détecteur MCP), la seconde au rayonnement filtré entre 20 et $80 \mathrm{~nm}$ (filtre en aluminium), zone d'intérêt pour l'amplification laser.
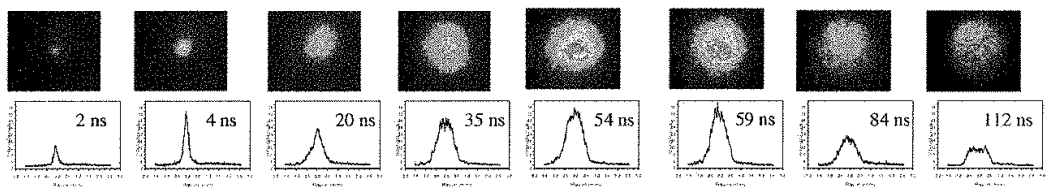

(a) Images non filtrées $(5-200 \mathrm{~nm})$
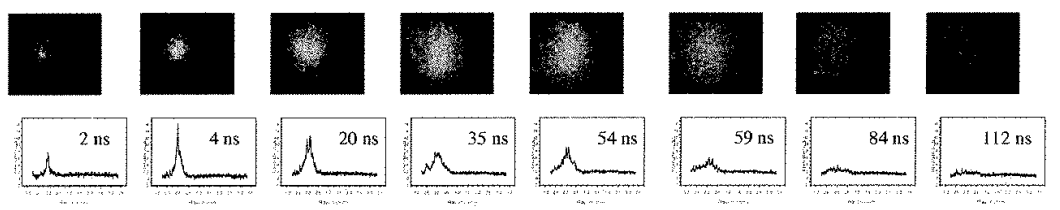

Figure 2 : images sténopé prises à différents instants de la décharge

La figure suivante (figure 3) résume l'évolution temporelle du rayon d'émission et de l'intensité totale émise (a), intégrée en deux dimensions sur les profils ci-dessus, et également 
de l'intensité émise le long de l'axe (b), pour la gamme spectrale 5-200 nm. Le rayon correspond à la demi-largeur à $1 / e^{2}$ d'une gaussienne ajustée aux profils d'émission ci-dessus.
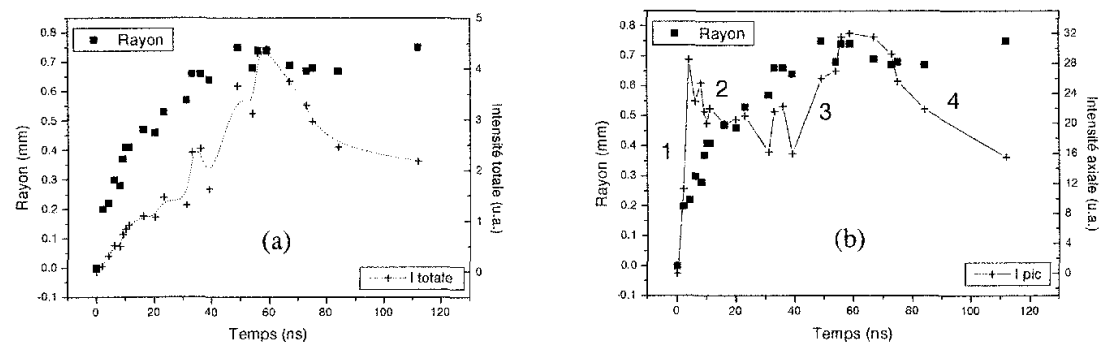

Figure 3 : évolution temporelle du rayon, de l'intensité totale (a) et axiale (b) d'émission (5-200 nm)

On observe quatre phases distinctes de l'évolution du plasma: (1) l'émission du plasma s'initie le long de l'axe, puis présente une surbrillance rapide; (2) l'expansion du plasma révélée par l'augmentation du rayon d'émission dans la gamme étendue 5-200 nm, entraîne une baisse d'intensité émise. (3) Lorsque l'expansion s'achève, le courant qui augmente chauffe le plasma et l'intensité, axiale ou totale, s'accroît jusqu'à un maximum simultané à celui du courant. Durant ces trois phases, l'intensité totale émise croît de façon monotone. Enfin, (4) les intensités totale et axiale diminuent à mesure que le courant décroît et que le plasma se refroidit. Le rayon reste alors inchangé. Sur les profils de la figure $2 . b$, on voit que l'intensité axiale correspondant à la gamme $20-80 \mathrm{~nm}$ présente un pic plus prononcé lors de la surbrillance que lors du maximum de courant. Les mesures filtrées montrent que le plasma émet efficacement dans la gamme d'intérêt dès les premiers instants de la décharge.

\section{DISCUSSIONS ET CONCLUSION}

On expose dans cette section les résultats des calculs effectués à partir du code, qui reproduisent qualitativement les observations. Par hypothèse, la densité de courant est supposée avoir un profil radial gaussien dont les paramètres dépendent du temps : la largeur varie linéairement avec le temps, selon une pente ajustée pour que le profil radial d'émission simulé se superpose au profil radial d'émission mesuré correspondant à chaque instant ; la hauteur est déduite de la valeur du courant mesuré expérimentalement. Le choix du profil gaussien est justifié par la forme des profils d'émission exposés sur la figure 2. La figure 4 montre l'évolution des intensités totale (a) et axiale (b) simulées, pour les domaines spectraux d'observation. D'autres types de profils pour la densité de courant n'ont pas permis de reproduire convenablement l'évolution de l'émission.
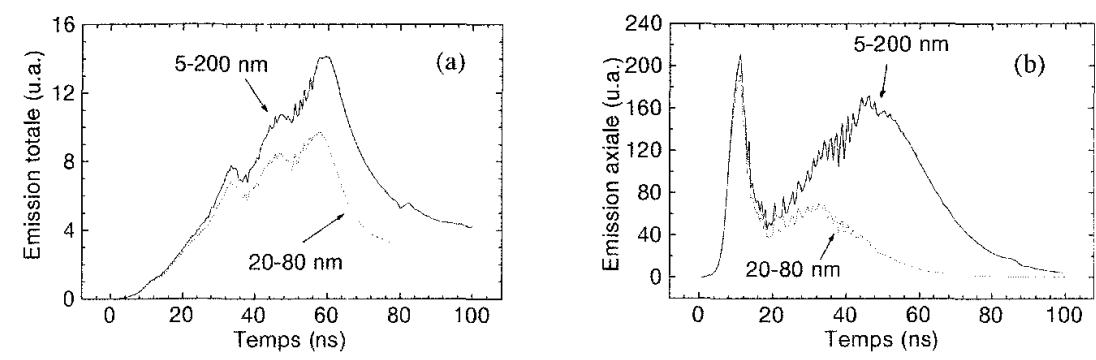

Figure 4 : évolution temporelle des intensités totale (a) et axiale (b) émises, calculées par le code 
L'évolution temporelle de l'émission est reproduite par les calculs, y compris la surbrillance qui suit l'initiation du plasma. La part relative du premier pic d'émission dans la gamme 20-80 $\mathrm{nm}$ par rapport au second pic est respectée. La densité électronique a été calculée au centre du plasma, ainsi que l'évolution temporelle de son profil radial. Le résultat est donné sur la figure 5 .
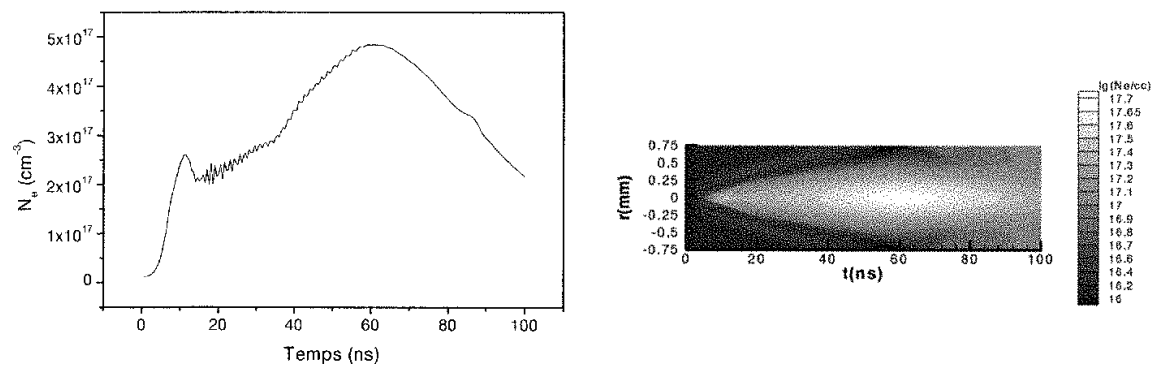

Figure 5: densité électronique calculée sur l'axe, et évolution temporelle de son profil radial

Le code reproduit correctement l'initiation axiale du plasma puis l'expansion radiale consécutive. Les calculs prédisent une densité électronique de l'ordre de quelques $10^{17} \mathrm{~cm}^{-3}$ au centre du plasma; en outre cette valeur correspond au maximum dans tout le plasma, c'està-dire que la densité électronique présente un profil radial convexe. D'après [3] et [9], ces conditions ne sont pas favorables à une amplification de la raie d' $\mathrm{Ar}^{8+}$ à $46,9 \mathrm{~nm}$ : en effet, la densité d'électrons est d'un ordre de grandeur trop faible pour favoriser le schéma de pompage envisagé [9], et d'autre part un profil convexe de densité entraîne des effets de réfraction néfastes à l'amplification sans cavité [3]. Des mesures de spectroscopie ont montré par ailleurs qu'aucune raie d' $\mathrm{Ar}^{8+} \mathrm{n}^{\prime}$ 'est émise durant la décharge, ce qui tend à confirmer ces résultats. L'expansion radiale du plasma observée durant la décharge est un facteur limitant l'augmentation de la densité, et donc peu favorable à l'effet recherché.

En conclusion, les expériences et simulations effectuées montrent que le dispositif employé dans les conditions de l'étude ne permet pas d'obtenir les conditions d'une émission cohérente de la raie à 46,9 . Bien que l'émission dans la gamme spectrale d'intérêt soit engendrée efficacement durant la décharge, l'initiation sur l'axe ne semble pas favoriser l'obtention d'un profil de densité électronique concave ayant le bon ordre de grandeur. Les recherches futures devront s'orienter vers l'obtention d'une compression magnétique efficace en vue de créer un plasma ayant la densité requise.

\section{Références}

1. R.C. Elton, in X-Ray Lasers (Academic Press, Boston, 1990), p. 99

2. J.J. Rocca, F.G. Tomasel, M.C. Marconi et al., Phys. Plasmas 2, 2547 (1995)

3. A. Ben-Kish, M. Shuker, R.A. Nemirovsky et al., Phys. Rev. Lett. 87, 015002-1 (2001)

4. G. Niimi, Y. Hayashi, M. Nakajima et al., J. Phys. D: Appl. Phys. 34, 2123 (2001)

5. G. Tomassetti, A. Ritucci, L. Palladino et al., Czech. J. Phys. 52, 405 (2002)

6. S. Götze, D. Hong, R. Dussart et al., J. Phys. IV France 11, Pr2-609 (2001)

7. L.C. Pitchford, J. Appl. Phys. 75, 7227 (1994)

8. I. Rutkevich, M. Mond, Y. Kaufman et al., Phys. Rev. E 62, 5603 (2000)

9. J.J. Rocca, Rev. Sci. Instrum. 70, 3799 (1999)

10. K. Lan, Y. Zhang, W. Zheng, Phys. Plasmas 6, 4343 (1999) 